\title{
Euglycemic ketoacidosis and sodium glucose co- transporter 2 inhibitors: is this novel class safe for the diabetic patients?
}

\begin{abstract}
Sodium glucose co-transporter 2 inhibitors are new class of drugs approved for the treatment of type 2 diabetes. U.S. Food and Drug Administration have recently announced that these approved drugs may cause euglycemic diabetic ketoacidosis. The exact mechanism of such event is still not known. Ongoing long-term clinical trials may be adding some more information about the safety of these newer agents.
\end{abstract}

Volume I Issue 4 - 2015

\author{
Hitesh Soni \\ Department of Physiology, University of Tennessee Health \\ Science Center, USA
}

Correspondence: Hitesh Soni, Department of Physiology, University of Tennessee Health Science Center, USA,Tel 9014481837,Email drhiteshsoni@gmail.com

Received: October 16,2015 | Published: October 27, 2015

Abbreviations: SGLT2i, sodium glucose co-transporter 2 inhibitors; T2D, type 2 diabetes; T1D, type 1 diabetes; USFDA, u.s. food and drug administration; euDKA, euglycemic diabetic ketoacidosis; FFAs, free fatty acids

\section{Introduction}

Sodium glucose co-transporter 2 inhibitors (SGLT2i) are novel class of antidiabetic agents developed primarily for the management of type 2 diabetes (T2D). These drugs lower plasma glucose by inhibition of glucose reabsorption from kidney, which finally increases glucose excretion in the urine. This action is insulin-independent and believed to be glucose-dependent. However, these drugs enhance glucose excretion even in glucose-demanding activities such as exercise, fasting or low carbohydrate diet. Very recently, the U.S. Food and Drug Administration (USFDA) warned that treatment with approved SGLT2i (canagliflozin, dapagliflozin and empagliflozin) may increase the risk of ketoacidosis with mild to moderate glucose elevations also known as euglycemic diabetic ketoacidosis (euDKA). Classically, DKA is one of the most frequent acute diabetic complications results from an absolute insulin deficiency and associated with a triad of uncontrolled hyperglycemia, metabolic acidosis and hyperketonemia. On contrary, euDKA is a condition which is associated with the use of SGLT2i but without marked hyperglycemia and this condition can be facilitated by food restriction, alcohol intake and inhibition of gluconeogenesis. ${ }^{1,2}$

\section{Potential mechanisms of SGLT2i-induced euDKA}

Clinical cases of DKA in SGLT2i-treated patients raise the question of how this novel class of approved drugs might lead to ketoacidosis (Figure 1)

Negative fluid and sodium balance: Normally, SGLT2 in the kidney lead to transport of glucose coupled with the energetically favored transport of sodium across the plasma membrane, which is dependent on concentration gradient and a membrane potential. Glucose in the circulation is constantly filtered in the kidneys and reabsorbed in the renal tubules via SGLT2. In the study state, sodium is also reabsorbed back by active transport. Inhibition of SGLT2 causes fluid and sodium imbalance and hypovolemia. Hypovolemic state leads to hormonal imbalance and elevates glucagon, cortisol, and epinephrine levels, which further increase insulin resistance, lipolysis, and euDKA. ${ }^{1}$

Hypoinsulinemia: SGLT2 $i$ are approved for the treatment of T2D. However, there is still investigational or off label use of SGLT2i to treat type 1 diabetes (T1D). It has been reported that in T1D patients there is a need to decrease the dose of insulin to reduce the risk of hypoglycemia. Decrease in circulating levels of insulin may enhance the lipolysis in adipose tissue and ketogenesis in the liver. Further, lower insulin-to-glucagon ratio in SGLT2i-treated T2D patients stimulates the process of lipolysis and ketogenesis. ${ }^{3,4}$

Hyperglucagonemia: There are two possible reasons for glucagon elevation in SGLT2i-treated patients, lower circulating levels of insulin and decreased SGLT2i-mediated glucose transport into pancreatic $\alpha$-cells. ${ }^{3}$ Recently, it has been demonstrated that SGLT2i induces hyperglucagonemia to compensate for the SGLT2i-mediated glucosuria. Elevated glucagon enhances proteolysis that contributes to reduction of fat mass, release of free fatty acids (FFAs) from the adipose tissue which might results in euDKA. ${ }^{5}$

Glucosuria: SGLT2i inhibit glucose reabsorption in the kidney and promote glucosuria, decrease plasma glucose, and induce moderate weight loss. Possibly, SGLT2i lower the renal threshold for glucose which leads to excretion of large amount of glucose in the urine. This process cause decrease in plasma glucose pool, which initiates the process of gluconeogenesis and FFAs from liver. This may lead to release of ketone bodies and euDKA when patients are on SGLT2i therapy for the treatment of T2D. ${ }^{1,4,5}$

Decrease in sodium reabsorption: It has been shown in animal study that non-selective inhibition of SGLT may increase sodium reabsorption in the renal tubule and therefore increase the carriermediated reuptake of negatively charged ketone bodies. This could be contributing factor for euDKA in SGLT2i-treated patients. ${ }^{4}$

Further investigation is required to elucidate the molecular mechanism of euDKA in SGLT2i-treated T1D and T2D patients. However, clinicians and patients need to consider the potential risk and remember the important points (Table 1) when prescribing and taking these novel medicines for the treatment of diabetes. Pre-renal 
azotemia due to volume depletion is a classic finding in DKA and it may progress to acute renal tubular necrosis. ${ }^{6}$ The more severe the volume depletion, the greater is the degree of renal impairment. However, there are no such reports available with the use of these novel agents. The ongoing long-term clinical trials will further reveal the necessary information on the safety of SGLT2i in both T1D and T2D.

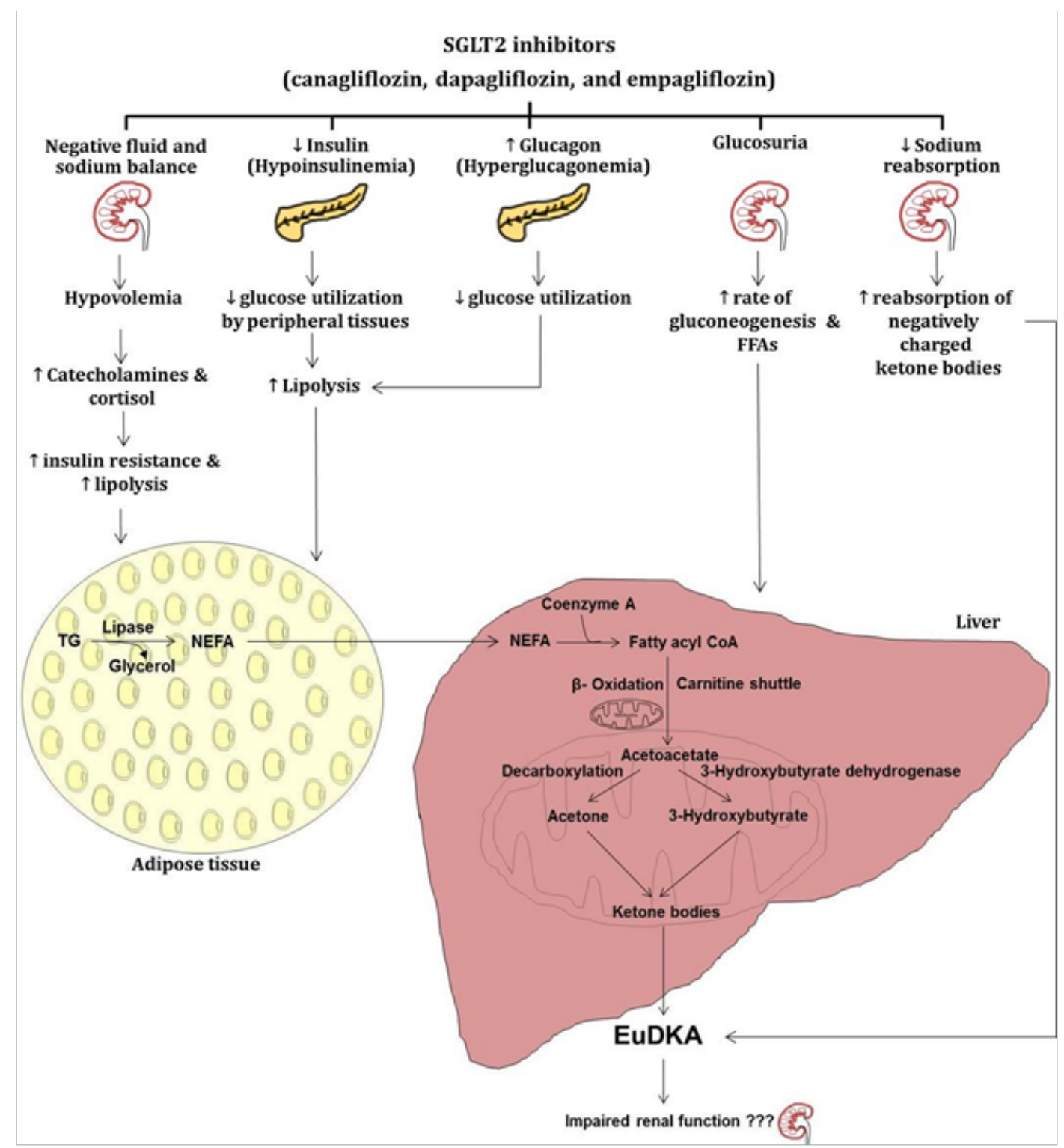

Figure I Potential mechanisms of SGLT2i-induced euDKA. EuDKA, Euglycemic Diabetic Ketoacidosis; FFAs, Free Fatty Acids; NEFA, Non Esterified Fatty Acids

Table I Important points to consider while using SGLT2i in type 2 diabetes.

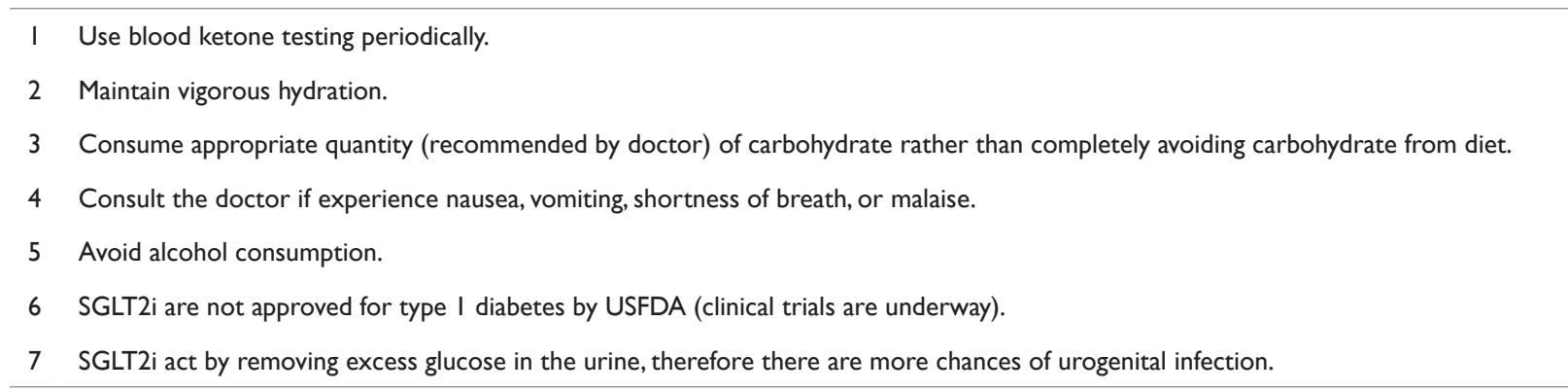




\section{Acknowledgements}

None.

\section{Conflict of interest}

The author declares no conflict of interest.

\section{References}

1. Peters AL, Buschur EO, Buse JB, et al. Euglycemic Diabetic Ketoacidosis: A Potential Complication of Treatment With SodiumGlucose Cotransporter 2 Inhibition. Diabetes Care. 2015;38(9):16871693.

2. Adrogue HJ, Eknoyan G, Suki WK. Diabetic ketoacidosis: role of the kidney in the acid-base homeostasis re-evaluated. Kidney Int. 1984;25(4):591-598.
3. Rosenstock J, Ferrannini E. Euglycemic Diabetic Ketoacidosis: A Predictable, Detectable, and Preventable Safety Concern with SGLT2 Inhibitors. Diabetes Care. 2015;38(9):1638-1642.

4. Taylor SI, Blau JE, Rother KI. SGLT2 Inhibitors May Predispose to Ketoacidosis. J Clin Endocrinol Metab. 2015;100(8):2849-2852.

5. Yabe D, NishikinoR, Kaneko M, et al. Short-term impacts of sodium/ glucose co-transporter 2 inhibitors in Japanese clinical practice: considerations for their appropriate use to avoid serious adverse events. Expert Opin Drug Saf. 2015;14(6):795-800.

6. Orban JC, Maizière EM, Ghaddab A, et al. Incidence and characteristics of acute kidney injury in severe diabetic ketoacidosis. PLoS One. 2014;9(10):e110925. 